\title{
13.
}

\section{Sur quelques propriétés des déterminants gauches.}

(Par Mr. A. Cayley de Cambridge.)

I.

Je donne le nom de déterminant gauche, à un déterminant formé par un système de quantités $\lambda_{r . s}$ qui satisfont aux conditions

(1) $\lambda_{r, s}=-\lambda_{s, r}[r \neq s]$.

J'appelle aussi un tel système, système gauche. On obtiendra des formules plus simples (quoique cette supposition ne soit pas essentielle), en considérant seulement les systèmes pour lesquels on a aussi

(2) $\lambda_{r . r}=1$.

Je suppose dans tout ce qui va suivre, que le déterminant est de l'ordre $n$, et que par conséquent les suffixes variables $r, s$ etc. s'étendent toujours depuis l'unité jusqu'à $\boldsymbol{n}$.

En posant les équations

(3) $\quad \Sigma_{r} \lambda_{r . s} x_{r}=\boldsymbol{P}_{s}, \quad \Sigma_{s} \lambda_{r, s} x_{s}=\boldsymbol{Q}_{r}$,

j'exprime les systèmes inverses qui déterminent les $\boldsymbol{P}, \boldsymbol{Q}$ par les $\boldsymbol{x}$, de la manière suivante:

(4) $\boldsymbol{K} x_{r}=\Sigma_{s} \Lambda_{r . s} \boldsymbol{P}_{s}$ et $\boldsymbol{K} \boldsymbol{x}_{s}=\Sigma_{r} \Lambda_{r . s} \boldsymbol{Q}_{r}$,

où $\boldsymbol{K}$ désigne le déterminant formé d'avec les quantités $\lambda_{r . s}$, et $\Lambda_{r . s}$ le coëfficient différentiel de $\boldsymbol{K}$ par rapport à $\lambda_{r . s}$; bien entendu, que la différentiation doit être effectuée avant d'avoir particularisé ces quantités par les équations (1), (2). On sait que ces fonctions $\Lambda$ satisfont aux conditions

$$
\text { (5) } \quad\left\{\begin{array}{l}
\Sigma_{r} \lambda_{r . s} \Lambda_{r . s^{\prime}}=0 \quad s \neq s^{\prime}, \\
\Sigma_{r} \lambda_{r . s} \Lambda_{r . s}=\boldsymbol{K}, \\
\Sigma_{r} \lambda_{r . s} \Lambda_{r^{\prime} s}=0 \quad r \neq \boldsymbol{r}^{\prime}, \\
\Sigma_{s} \lambda_{r . s} \Lambda_{r . s}=\boldsymbol{K} .
\end{array}\right.
$$

Je tiré des équations (4), en échangeant $r$ et $s$ dans la dernière de ces équations:

$$
\text { (6) } \Sigma_{s} \mathcal{A}_{r . s} \boldsymbol{P}_{s}=\Sigma_{s} \mathcal{A}_{s, r} \boldsymbol{Q}_{s} \text {. }
$$

Et de là, en multipliant respectivement les différentes équations de ce système par $\lambda_{s^{\prime}, r}$, et prenant la somme de ces produits:

$$
\Sigma_{s}\left(\Sigma_{r} \lambda_{s^{\prime}, r} \Lambda_{r, s}\right) \boldsymbol{P}_{s}=\Sigma_{s}\left(\Sigma_{r} \lambda_{s^{\prime}, r} \Lambda_{s, r}\right) \boldsymbol{Q}_{s}
$$


On a d'abord par les équations (5)

$$
\text { (8) } \quad \Sigma_{s}\left(\Sigma_{r} \lambda_{s^{\prime}, r} \Lambda_{s . r}\right) \boldsymbol{Q}_{s}=\boldsymbol{K} \boldsymbol{Q}_{s^{\prime}}
$$

Puis par les équations (1 et 2)

$$
\text { (9) } \quad \Sigma_{r} \lambda_{s^{\prime} . r} \Lambda_{r . s}=2 \Lambda_{s^{\prime}, s}-\Sigma_{r} \lambda_{r . s^{\prime}} \Lambda_{r . s} \text {, }
$$

c'est-à-dire par les équations (5):

ce qui donne

$$
\left\{\begin{array}{l}
\Sigma_{r} \lambda_{s^{\prime}, r} \Lambda_{r . s}=2 \Lambda_{s^{\prime}, s} s^{\prime} \neq s \text { et } \\
\Sigma_{r} \lambda_{s^{\prime}, r} \Lambda_{r . s^{\prime}}=2 \Lambda_{s^{\prime}, s^{\prime}}-K
\end{array}\right.
$$

$$
\text { (11) } \Sigma_{s}\left(\Sigma_{r} \lambda_{s^{\prime}, r} \Lambda_{r . s}\right) \boldsymbol{P}_{s}=2\left(\Sigma_{s} \Lambda_{s^{\prime}, s} \boldsymbol{P}_{s}\right)-\boldsymbol{K P _ { s ^ { \prime } }}
$$

Substituant les équations ( 8 et 11) dans la formule ( 7 ), on obtiendra, en écrivant $r$ au lieu de $s^{\prime}$ :

et également

$$
\boldsymbol{K} \boldsymbol{Q}_{r}=2\left(\Sigma_{s} A_{r . s} \boldsymbol{P}_{s}\right)-\boldsymbol{K} \boldsymbol{P}_{r}
$$

$$
\boldsymbol{K} \boldsymbol{P}_{s}=2\left(\Sigma_{r} \Lambda_{r . s} \boldsymbol{Q}_{r}\right)-\boldsymbol{K} \boldsymbol{Q}_{s}
$$

Posant maintenant

$$
\left\{\begin{array}{l}
K \alpha_{r . s}=2 \Lambda_{r . s} r \neq s \text { et } \\
K \alpha_{r . r}=2 \Lambda_{r . r}-K
\end{array}\right.
$$

les formules (12), (13) se changeront en

$$
\text { (15) } \boldsymbol{Q}_{r}=\Sigma_{s} \alpha_{r . s} \boldsymbol{P}_{s} \text { et } \boldsymbol{P}_{s}=\Sigma_{r} \alpha_{r . s} \boldsymbol{Q}_{r} \text { : }
$$

équations qui sont nécessairement équivalentes. On a done identiquement

$$
\left\{\begin{array}{l}
\text { (1) }\left\{\begin{array} { l } 
{ \Sigma _ { r } \alpha _ { r . s } \alpha _ { r . s ^ { \prime } } = 0 \quad s \neq s ^ { \prime } , } \\
{ \Sigma _ { r } \alpha _ { r . s } \alpha _ { r . s } = 1 , }
\end{array} \text { (2) } \left\{\begin{array}{l}
\Sigma_{s} \alpha_{r . s} \alpha_{r^{\prime} s}=0 \\
\Sigma_{s} \alpha_{r . s} \alpha_{r . s}=1:
\end{array} \quad r \neq r^{\prime},\right.\right.
\end{array}\right.
$$

c'est-à-dire, on a trouvé un système de $\left(n^{2}\right)$ quantités $\alpha_{r_{0} s}$, fonctions explicites et rationnelles d'un nombre $\frac{1}{2} n(n-1)$ de variables indépendantes, qui satisfont identiquement aux formules $(16,1)$ ou $(16,2)$. On sait qu'en géométrie cela veut dire que pour $n=2$ ou $n=3$ de tels systèmes donnent les coëfficients propres à effectuer la transformation de deux systèmes de coordonnées rectangulaires; nous dirons par analogie, que des systèmes qui satisfont aux équations (16,) pour une valeur quelconque de $n$, sont propres à effectuer la transformation entre deux systèmes de coordonnées rectangulaires. On a donc le théorème suivant:

Les coëfficients propres à la transformation de coordonnées rectangulaires, peuvent être exprimés rationnellement au moyen de quantités ar- 
bitraires $\lambda_{r . s}$, soumises aux conditions

$$
\lambda_{r, s}=-\lambda_{s, r}[r \neq s] ; \quad \lambda_{r . r}=1 .
$$

Pour les développer, il faut d'abord former le déterminant $K$ de ce système, puis le système inverse $\Lambda_{r . s}, \ldots$ et écrire

$$
K \alpha_{r, s}=2 \Lambda_{r . s}[r \neq s] ; \quad K \alpha_{r, r}=2 \Lambda_{r, r}-K ;
$$

ce qui donne le système cherché.

Soit par exemple $n=3$. Écrivons pour le système des quantités $\lambda_{r . s}$ :

$$
\left\{\begin{array}{r}
1, \quad \nu,-\mu, \\
-\nu, \quad 1, \quad \lambda, \\
\mu,-\lambda, \quad 1,
\end{array}\right.
$$

ce qui donne $\boldsymbol{K}=1+\lambda^{2}+\mu^{2}+v^{2}$, et pour le système des fonctions $\boldsymbol{A}_{r . s}$

$$
\left\{\begin{array}{rrr}
1+\lambda^{2}, & \lambda \mu+\nu, & \nu \lambda-\mu, \\
\lambda \mu-\nu, & 1+\mu^{2}, & \mu \nu+\lambda, \\
\nu \lambda+\mu, & \mu \nu-\lambda, & 1+\nu^{2} .
\end{array}\right.
$$

Delà on obtient pour le système de coëfficients $\alpha, \beta, \gamma ; \alpha^{\prime}, \beta^{\prime}, \gamma^{\prime} ; \alpha^{\prime \prime}, \beta^{\prime \prime}, \gamma^{\prime \prime}$ :

$$
\left\{\begin{array}{lll}
K \alpha=1+\lambda^{2}-\mu^{2}-\nu^{2}, & K \alpha^{\prime}=2(\lambda \mu+\nu), & K \alpha^{\prime \prime}=2(\nu \lambda-\mu), \\
K \boldsymbol{K} \beta=2(\lambda \mu-\nu), & \boldsymbol{K} \beta^{\prime}=\left(1+\mu^{2}-\nu^{2}-\lambda^{2}\right), & \boldsymbol{K} \beta^{\prime \prime}=2(\mu \nu+\lambda), \\
\boldsymbol{K} \gamma=2(\nu \lambda+\mu), & \boldsymbol{K} \gamma^{\prime}=2(\mu \nu-\lambda), & \boldsymbol{K} \gamma^{\prime \prime}=\left(1+\nu^{2}-\lambda^{2}-\mu^{2}\right)
\end{array}\right.
$$

ce qui se rapporte à la transformation

$$
\begin{cases}x=\alpha x_{1}+\beta y_{1}+\gamma z_{1}, & x_{1}=\alpha x+\alpha^{\prime} y+\alpha^{\prime \prime} z, \\ y=\alpha^{\prime} x_{1}+\beta^{\prime} y_{1}+\gamma^{\prime} z_{1}, & y_{1}=\beta x+\beta^{\prime} y+\beta^{\prime \prime} z, \\ z=\alpha^{\prime \prime} x_{1}+\beta^{\prime \prime} y_{1}+\gamma^{\prime \prime} z_{1} & z_{1}=\gamma x+\gamma^{\prime} y+\gamma^{\prime \prime} z,\end{cases}
$$

de deux systèmes de coordonnées rectangulaires. En effet, les coëfficients $\lambda, \mu, \nu$ ont une signification géométrique: Les axes $A x_{1}, A y_{1}, A z_{1}$ vont coïncider avec les axes $\boldsymbol{A} x, \boldsymbol{A} y, \boldsymbol{A} z$, par la rotation $\theta$ autour d'un certain axe $\boldsymbol{A R}$ (qu'on peut nommer "Axe résultante"). En prenant $f, g, h$ pour les inclinaisons de cet axe à $\boldsymbol{A} x, A y, A z$, on a $\lambda=\operatorname{tang} \frac{1}{2} \theta \cos f, \mu=\operatorname{tang} \frac{1}{2} \theta \cos g$, $\nu=\operatorname{tang} \frac{1}{2} \theta \cos h$. Cette expression de l'axe $\boldsymbol{A R}$ est due à Euler; les quantités $\lambda, \mu, \nu$ ont été introduites pour la première fois, par M. Olinde Rodrigues, dans un mémoire „Sur les lois géométriques qui regissent les déplacements d'un système solide" (Liouville Tome V.), où il donne les expressions qu'on vient de trouver ici, pour les coëfficients de la transformation, en termes de $\lambda, \mu, \nu$. Ces mêmes quantités $\lambda, \mu, \nu$. (il y a à remarquer cela en passant) sont liées de la manière la plus étroite avec celles de la belle théorie de Sir $W$. Hamilton 
sur les Quaternions. Je les ai appliquées à la théorie de la rolation d'un corps solide. Avant de donner une idée des résultats auxquels je suis parvenu, je passe aux formules de transformation qui se rapportent au cas de $n=4$. Je prends ici pour le système des quantités $\lambda$ :

$$
\left\{\begin{array}{r}
1, \quad a, \quad b, \quad c, \\
-a, \quad 1,-h, \quad g \\
-b, \quad h, \quad 1,-f \\
-c,-g, \quad f, \quad 1,
\end{array}\right.
$$

ce qui donne, en mettant pour abréger $a f+b g+c h=\theta$,

$$
K=1+a^{2}+b^{2}+c^{2}+f^{2}+g^{2}+h^{2}+\theta^{2},
$$

et puis pour les quantités $\Lambda_{r . s}$ le système

$$
\left\{\begin{array}{rrrr}
1+f^{2}+g^{2}+h^{2}, & f \theta+a+b h-c g, & g \theta+b+c f-a h, & h \theta+c+a g-b f \\
-f \theta-a+b h-c g, & 1+f^{2}+b^{2}+c^{2}, & -c \theta-h+f g-a b, & b \theta+g-h f-c a, \\
-g \theta-b+c f-a h, & c \theta+h+f g-a b, & 1+g^{2}+c^{2}+a^{2}, & -a \theta-f+g h-b c, \\
-h \theta-c+a g-b f, & -b \theta-g+h f-c a, & a \theta+f+g h-b c, & 1+h^{2}+a^{2}+b^{2},
\end{array}\right.
$$

de manière que pour

$$
\left\{\begin{array}{llll}
\boldsymbol{K} \alpha, & \boldsymbol{K} \alpha^{\prime}, & \boldsymbol{K} \alpha^{\prime \prime}, & \boldsymbol{K} \alpha^{\prime \prime \prime}, \\
\boldsymbol{K} \beta, & \boldsymbol{K} \beta^{\prime}, & \boldsymbol{K} \beta^{\prime \prime}, & \boldsymbol{K} \beta^{\prime \prime \prime}, \\
\boldsymbol{K} \gamma, & \boldsymbol{K} \gamma^{\prime}, & \boldsymbol{K} \gamma^{\prime \prime}, & \boldsymbol{K} \gamma^{\prime \prime \prime}, \\
\boldsymbol{K} \delta, & \boldsymbol{K} \delta^{\prime \prime}, & \boldsymbol{K} \delta^{\prime \prime \prime}, & \boldsymbol{K} \delta^{\prime \prime \prime},
\end{array}\right.
$$

on obtient le système suivant:

$$
\left\{\begin{array}{llll}
1+f^{2}+g^{2}+h^{2}-a^{2}-b^{2}-c^{2}, 2(f \theta+a+b h-c g), & 2(g \theta+b+c f-a h), & 2(h \theta+c+a g-b f), \\
2(-f \theta-a+b h-c g), & 1+f^{2}+b^{2}+c^{2}-g^{2}-h^{2}-a^{2}, & 2(-c \theta-h+f y-a b), & 2(b \theta+g-h f-c a), \\
2(-g \theta-b+c f-a h), & 2(c \theta+h+f f-a b), & \left(1+g^{2}+c^{2}+a^{2}-f^{2}-h^{2}-b^{2}\right), & 2(-a \theta-f+g h-b c), \\
2(-h \theta-c+a g-b f), & 2(-b \theta-g+h f-c a), & 2(a \theta+f+g h-b c), & \left(1+h^{2}+a^{2}+b^{2}-f^{2}-g^{2}-c^{2}\right),
\end{array}\right.
$$

et ainsi de suite pour des valeurs quelconques de $n$.

\section{II.}

Maintenant je vais citer les formules que j'ai présentées dans le Journal de Cambridge Tome III. p. 225, pour la rotation d'un corps solide autour d'un point fixe. Mettant, comme à l'ordinaire les vitesses angulaires autour des axes principaux $=p, q, r$; les moments du corps pour ces mêmes axes $=A, B, C$ et la fonction des forces $=V$ : les équations citées pourront ètre écrites sous la forme

$$
d t=\frac{d p}{P}=\frac{d q}{Q}=\frac{d r}{R}=\frac{d \lambda}{\Lambda}=\frac{d \mu}{M}=\frac{d \nu}{N},
$$


où

$$
\begin{aligned}
& \int \boldsymbol{P}=\frac{1}{A}\left((B-C) g r+\frac{1}{2}\left\{\left(1+\lambda^{2}\right) \frac{d V}{d \lambda}+(\lambda \mu+\nu) \frac{d V}{d \mu}+(\lambda \nu-\mu) \frac{d V}{d \nu}\right\}\right), \\
& \left\{\begin{array}{l}
\boldsymbol{Q}=\frac{1}{\boldsymbol{B}}\left((\boldsymbol{C}-\boldsymbol{A}) \boldsymbol{r p}+\frac{1}{2}\left\{(\mu \lambda-\nu) \frac{d V}{d \lambda}+\left(1+\mu^{2}\right) \frac{d V}{d \mu}+(\mu \nu+\lambda) \frac{d V}{d \nu}\right\}\right), \\
\boldsymbol{R}=\frac{1}{C}\left((A-B) p q+\frac{1}{2}\left\{(\nu \lambda+\mu) \frac{d V}{d \lambda}+(\mu \nu-\lambda) \frac{d V}{d \mu}+\left(1+\nu^{2}\right) \frac{d V}{d \nu}\right\} !\right),
\end{array}\right. \\
& \Lambda=\frac{1}{2}\left(\left(1+\lambda^{2}\right) \boldsymbol{p}+(\lambda \mu-\nu) \boldsymbol{y}+(\lambda \nu+\mu) \boldsymbol{r}\right) \\
& M=\frac{1}{2}\left((\mu \lambda+\nu) p+\left(1+\mu^{2}\right) \boldsymbol{q}+(\mu \nu-\lambda) r\right), \\
& N=\frac{1}{2}\left((\nu \lambda-\mu) p+(\nu \mu+\lambda) \boldsymbol{q}+\left(1+\nu^{2}\right) r\right) \text {. }
\end{aligned}
$$

En effet, pour obtenir ces formules, il n'y a qu'à chercher au moyen de $\lambda, \mu, \nu$ et de leurs derivées par rapport aux temps $\lambda^{\prime}, \mu^{\prime}, \nu^{\prime}$ l'expression de la fonction $\boldsymbol{T}=\frac{1}{2}\left(\boldsymbol{A} \boldsymbol{p}^{2}+\boldsymbol{B} \boldsymbol{q}^{2}+\boldsymbol{C r}^{2}\right)$ [qui exprime la demi-somme des forces vives]: cela fait, les formules générales que Lagrange a données pour la solution des problèmes de dynamique, conduisent immédiatement aux équations en question. Dans le mémoire cité j'ai intégré ces équations pour le cas ou la fonction $\boldsymbol{V}$ est zéro, et en prenant, comme dans la théorie ordinaire, le plan invariable pour plan des deux axes. Ce n'est que dernièrement que j'ai trouvé la manière convenable do trailer ce système d'équations; je le fais au moyen de deux nouvelles variables $\Omega, v$, entre lesquelles je trouve une équation différentielle dont les variables sont séparées, et j'exprime en termes de celles-ci les autres variables du problème, y compris le temps; et cela sans aucune supposition particulière, relative a la position des axes des coordonnées par rapport au plan invariable. Le developpement de cette théorie paraitra dans le prochain $\mathrm{N}^{\circ}$. du "Cambridge et Dublin Mathématical journal." Je m'occupe aussi de la recherche des formules pour les variations des constantes arbitraires relatives aux forces perturbatrices. Il serait bien intéressant (comme problème d'analyse pure) d'étendre ces recherches au cas d'une valeur quelconque de $n$; il faudrait pour cela, chercher les valeurs des quantités analogues à $p, q, r$, former une fonction $\boldsymbol{T}$, en prenant la somme des carrés de chacune de ces quantités, chaque "carré multiplié par un coëfficient constant, et puis former les équations $\frac{d}{d t} \cdot \frac{d T}{d \lambda^{\prime}}-\frac{d T}{d \lambda}=0$ etc., analogues aux équations de Dynamique. Mais je n'ai encore rien trouvé sur ce sujet.

Cambridge $24^{\text {iène }}$ Févr. 1846. 